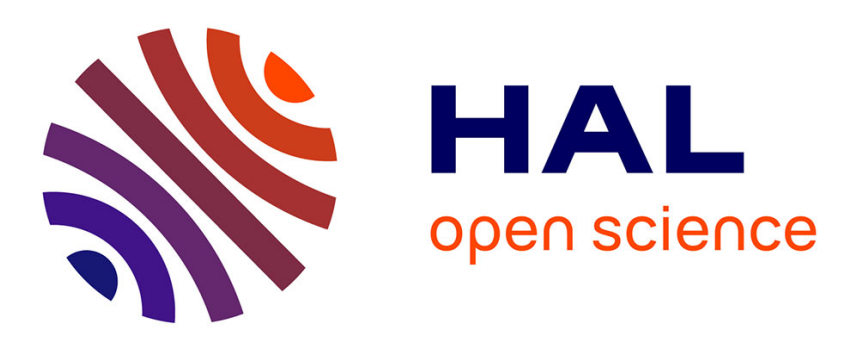

\title{
Full-Duplex Efficient Channel Codes for Residual Self-Interference/Quantization Noise Cancellation
}

Bao Quoc Vuong, Roland Gautier, Anthony Fiche, Mélanie Marazin

\section{To cite this version:}

Bao Quoc Vuong, Roland Gautier, Anthony Fiche, Mélanie Marazin. Full-Duplex Efficient Channel Codes for Residual Self-Interference/Quantization Noise Cancellation. International Conference on Signal Processing and Communication Systems (ICSPCS), Dec 2021, Sydney, Australia. hal03481171

\author{
HAL Id: hal-03481171 \\ https://hal.science/hal-03481171
}

Submitted on 15 Dec 2021

HAL is a multi-disciplinary open access archive for the deposit and dissemination of scientific research documents, whether they are published or not. The documents may come from teaching and research institutions in France or abroad, or from public or private research centers.
L'archive ouverte pluridisciplinaire HAL, est destinée au dépôt et à la diffusion de documents scientifiques de niveau recherche, publiés ou non, émanant des établissements d'enseignement et de recherche français ou étrangers, des laboratoires publics ou privés. 


\title{
Full-Duplex Efficient Channel Codes for Residual Self-Interference/Quantization Noise Cancellation
}

\author{
Bao Quoc Vuong, Student Member, IEEE, Roland Gautier, Member, IEEE, Anthony Fiche, Member, IEEE \\ and Mélanie Marazin, Member, IEEE \\ Univ Brest, Lab-STICC, CNRS, UMR 6285, F-29200 Brest, France \\ \{bao.vuong, roland.gautier, anthony.fiche and melanie.marazin\}@univ-brest.fr
}

\begin{abstract}
Full-Duplex (FD) systems are became very attractive technique for 5G and beyond transmissions by offering higher spectral efficiency. The implementation of a real FD system can be a challenging task due to the analog and residual Self-Interference (SI) and some imperfections introduced by analog components such as quantization error of Digital-toAnalog/ Analog-to-Digital Converters (DAC/ADC). This paper investigates in the digital domain different channel coding schemes to compensate the residual SI and quantization noise cancellation process in case of Single Input Single Output (SISO) FD transmission. The promising channel coding schemes from the Third Generation Partnership Project (3GPP) such as 5G Quasi-Cyclic Low Density Check (QC-LDPC), 5G Polar Codes and LTE Turbo codes have been considered. Several numerical simulations are performed to evaluate the Bit Error Rate (BER) performance.
\end{abstract}

Index Terms-Full-Duplex, Digital self-interference cancellation, Recursive least square, Channel coding schemes, 5G transmission systems, Quantizer device architecture

\section{INTRODUCTION}

In the future, the rapid growth of wireless and broadband communication requires higher spectral efficiency to account for the higher rate, lower latency transmission and maintain high level of security when we move toward to 5G standard. Furthermore, confidentiality in wireless medium becomes more important to not only protect the legitimate information but also improve the system's performance [1]. The use of FD transmission, with good SI control, may be considered as a first level of data security in 5G and beyond, by transmitting simultaneously the useful signal and the jamming signal. Therefore, the spectral efficiency is doubled when compared with the traditional Half-Duplex (HD) method, and the received signal will be more difficult to exploit by the eavesdroppers [2]. However, the problem of SI phenomenon is the main challenge of FD transmission, which could be up to $120 \mathrm{~dB}$ in power when the system suffers from strong SI environment [3]. In order to overcome this problem, several techniques have been studied such as RF cancellation technique [4], analog cancellation and digital cancellation [5].

Moreover, the authors in [6] showed that channel coding, such as QC-LDPC codes and Polar codes are considered as potential candidates to ensure the fiability of information for $5 \mathrm{G}$ and beyond communication systems. The development of $5 \mathrm{G}$ will lead to an expansion of the network by providing the platform to connect a huge number of mobile devices together, which requires novel fundamental security approach. Among the error correcting codes, LDPC codes [7], Polar codes [8] and Turbo codes [9] are the most efficient techniques because they can achieve nearly Shannon capacity performance. The 3GPP project has introduced the QC-LDPC and Polar codes as the standard codes of $5 \mathrm{G}$ for the control information in uplink and downlink because they support for a number of lifting size and different code rate with high throughput and low latency [10]. Furthermore, Turbo codes are the fundamental channel coding technique in $3 \mathrm{G}$, 4G Long Term Evolution (LTE) system and Machine Type Communications (MTC) or Machine to Machine (M2M) communications [11]. It can be also considered as a candidate in $5 \mathrm{G}$ channel coding scheme for short length frame with lower processing throughput for the massive Machine Type Communication (mMTC) or massive Machine to Machine (mM2M) [12].

Last but not least, the quantization noise, which is produced by the quantization process performed by DAC at the transmitter and ADC at the receiver, is also a main factor that impacts to the FD transmission system performances [13]. The quantization bit resolution and the dynamic range should be chosen carefully based on the requirement of the sampling rate and the applications. Many DAC/ADC transceiver devices have been designed and launched out to the market for $4 \mathrm{G}$, $5 \mathrm{G}$ and also IoT applications. For example, the LTC2000 DAC family [14] uses 11-16 bits quantization resolution for LTE MIMO and 5G transmissions at the transmitter. While at the receiver, the 12 bits $\Sigma \Delta$ AD9361 [15] is used in ADC process of $4 \mathrm{G}$ and $5 \mathrm{G}$ transmission. DAC/ADC process is an important part of any communication system, therefore, it is necessary to take into account the problem of residual quantization noise of DAC/ADC process in FD transmission.

So, in this paper, we investigate in digital domain the channel coding schemes in case of Single Input Single Output (SISO) FD transmission to overcome the effects of residual SI and quantization noise cancellation process. The Digital Self-Interference Cancellation (DSIC) process is based on Recursive Least Square (RLS) algorithm, because it has a better result when compared with others such as Least Mean Square (LMS), Normalized Least Mean Squared (NLMS) [5], [16]. First, we illustrate the influence of quantization noise to DSIC process and compare the effects of SI channel power to SISO FD transmission system with and without DSIC. Then, BER performance obtained with and without DAC/ADC process will be illustrated by changing the quantization bit 
resolution at DAC/ADC process. Next, the performances of different quantizer device architectures at the receiver such as $\Sigma \Delta$ [17], Gaussian with mid-rise [18], pipeline [19] and Successive Approximation Registers ADC (SAR) [20] are evaluated. Then, a comparison between the system with and without DAC/ADC for both case of HD and FD transmission is considered. Finally, the choice of bit resolution for DAC/ADC process is also noticed. For the rest of the paper, the system without DAC/ADC process is called ideal case.

The remaining of this paper is organized as follow. Section II describes briefly the model of SISO FD transmission system with DSIC process, the channel coding process, as well as the DAC/ADC process. Simulation specifications and results are presented in Section III. Finally, some highlight conclusions are given in Section IV.

\section{System Model AND PROBlem Formulation}

\section{A. System Model and DSIC Process}

The general SISO FD transmission model between two users $\mathrm{A}$ and $\mathrm{B}$ in the presence of channel coding scheme, DSIC and DAC/ADC process is described in Fig. 1.

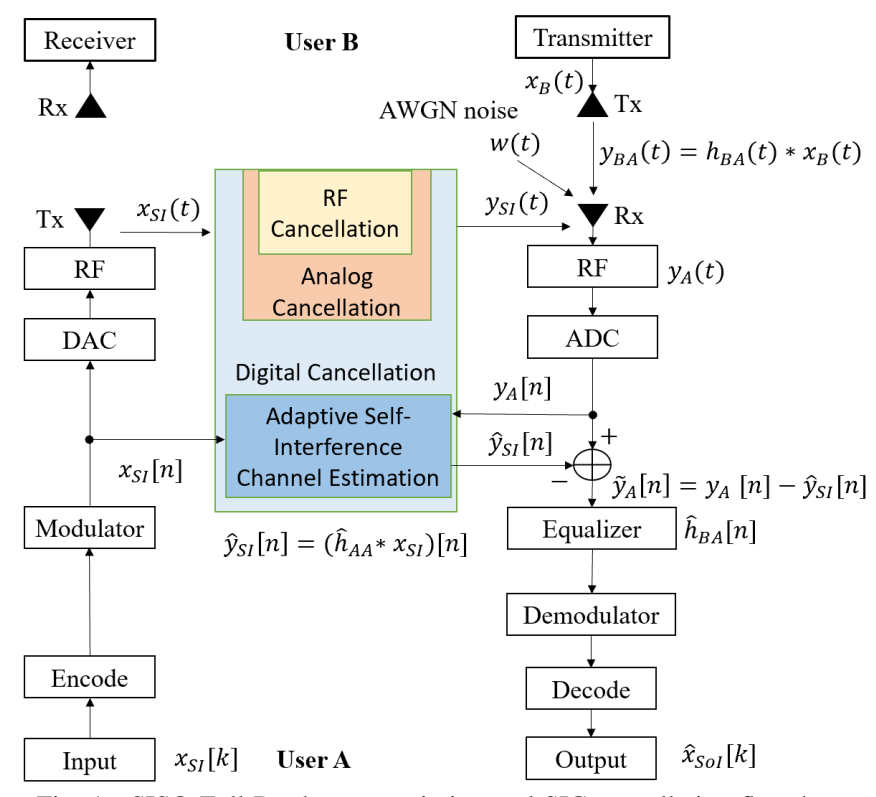

Fig. 1. SISO Full-Duplex transmission and SIC cancellation flowchart.

At the transmitter of user A, the digital input signal $x_{S I}[k]$ is encoded, modulated by using QPSK modulator and then transmitted through DAC converter. Then it turns into RF transmitting signal and is sent by transmitting antenna Tx to the user $\mathrm{B}$, which denotes as $x_{S I}(t)$. We have the same process at the transmitter of user B to obtain the transmitting signal of interest $x_{B}(t)$ and it will be transmitted through the multi-path fading channel environment with Additive White Gaussian Noise (AWGN) with $\mathcal{C N}\left(0, \sigma_{w}^{2}\right)$ from the environment.

At the receiver of user $A$, the receiving signal consists of several signals including the signal of interest $y_{B A}(t)$ from user B transmitter, the SI signal $y_{S I}(t)$ from the transmitter of user A itself and the complex Additive White Gaussian Noise (AWGN) $w(t)$ with $\mathcal{C N}\left(0, \sigma_{w}{ }^{2}\right)$. It can be given as:

$$
\begin{aligned}
y_{A}(t) & =y_{S I}(t)+y_{B A}(t)+w(t) \\
& =h_{A A}(t) * x_{S I}(t)+h_{B A}(t) * x_{B}(t)+w(t)
\end{aligned}
$$

where $h_{B A}(t), h_{A A}(t)$ are the intended fading channel and the SI channel, respectively ( $*$ is the convolution operation).

In FD transmission, the SI channel consists of two components: line-of-sight (LoS), which is generated by the direct link between transmitter and receiver and non line-of-sight (NLoS), which is produced by the signal scatters. So, its first tap could be modeled as Rician fading and the remaining channel taps are modeled as Rayleigh fading [5]. The LoS component is cancelled by RF cancellation and analog cancellation processes, and the remain residual component will be cancelled in digital domain [21]. In our work, we focus mainly on the digital domain to cancel the residual SI signal after RF and analog SI cancellation. So, a multi-path timeinvariant channel model with Rayleigh fading distribution is applied for both intended channel $h_{B A}$ and SI channel $h_{A A}$ with $\mathcal{C N}\left(0, \sigma_{h_{B A}}^{2}\right)$ and $\mathcal{C N}\left(0, \sigma_{h_{A A}}^{2}\right)$, respectively. The SI channel is fixed with 3 taps under the assumption that any LoS component is efficiently reduced by antenna isolation and the major effect comes from scattering. These channel models are independent in each transmission frame. The intended channel $h_{B A}$ is modeled according to the ITU-R Pedestrian test environment channel model [22].

The received signal will pass through ADC process to convert to discrete time domain, denoted as $y_{A}[n]$. Then, a copy version of the transmitted signal $x_{S I}[n]$ in digital domain is used as the reference signal to eliminate the SI component by using RLS algorithm, which is given in [23]. As a result, we can effectively obtain the estimation of the SI channel $\hat{h}_{A A}[n]$. So, the output signal can be expressed as:

$$
\begin{array}{r}
\tilde{y}_{A}[n]=y_{A}[n]-\hat{y}_{S I}[n]=y_{B A}[n]+y_{S I}[n]-\hat{y}_{S I}[n]+w[n] \\
=\left(h_{B A} * x_{B}\right)[n]+\left(h_{A A} * x_{S I}\right)[n]-\left(\hat{h}_{A A} * x_{S I}\right)[n]+w[n]
\end{array}
$$

Then, the residual signal $\tilde{y}_{A}[n]$ will go to the Decision Feedback Equalizer (DFE) [24] in order to reconstruct the input signal of user B and reduce the effects of multi-path fading channel and AWGN. Finally, the signal will pass through the demodulation block and the decoding block to obtain the estimated signal of interest from user B $\hat{x}_{S o I}[k]$.

\section{B. Channel coding schemes}

The construction of channel encoding and decoding process is shown in Fig 2. In $5 \mathrm{G}$ network and its applications, a Cyclic Redundancy Check (CRC) bit sequence is added to the initial information bits to form a bit sequence with length of $K$. Then, they will be encoded to obtain the codeword sequence with length $E$ by using three channel coding schemes proposed by $3 \mathrm{GPP}$ project such as 5G LDPC codes, 5G Polar codes [25] and LTE Turbo codes [11]. To adapt the code rate $R=K / N$, the rate matching including bit shortening, 
bit puncturing or bit repeating should be applied to create a codeword with length $N$ [10], [11], [26]. At the decoder, the Log-Likelihood-Ratio (LLR) is used to reduce the calculation complexity by replacing a very large number of multiplications by summation in log-domain as in [27]. The LLR belief information containing $N$ symbols received from the channel and demodulation process will be passed through the rate recovering process to reconstruct the message sequence with length $E$ [10], [11], [26]. The Sum-Product Algorithm (SPA) [28], Successive Cancellation List Decoding with CRC Aided (SCLD-CA) [29] and Max-Log Maximum A Posteriori (MaxLog-MAP) algorithm [30] are used in the decoding process for 5G LDPC codes, 5G Polar codes and LTE Turbo codes, respectively. After that, based on $\mathrm{CRC}$ attachment, the estimate message bits are achieved.

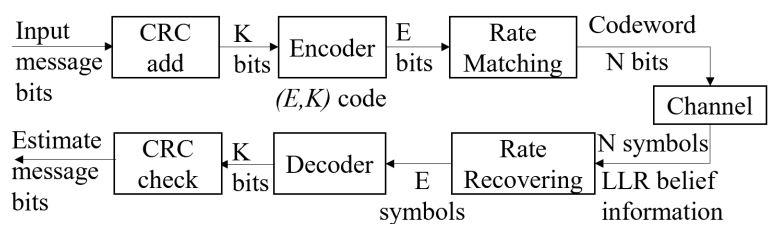

Fig. 2. Channel coding process

\section{DAC/ADC process}

The DAC process at the transmitter is presented in Fig. 3. The QPSK symbols with double complex number will be splitted into 2 separated components: in-phase (I) and quadrature (Q), each component then passes independently through the up sample with the factor $m_{u p}$ and then goes through DAC quantizer with the bit resolution $B_{D A C}$, the dynamic voltage range $V_{D A C}$ before going to the FIR interpolating filter to convert these discrete values to the continuous values and send to the wireless fading environment.

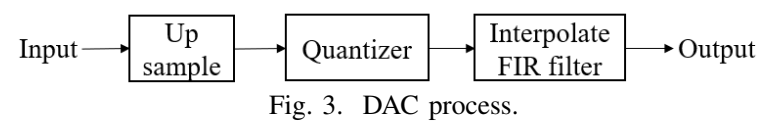

At the receiver as described in Fig. 4, the I and Q components that are received from the channel will pass through different types of ADC such as $\Sigma \Delta$, Gaussian with mid-rise, pipeline as well as SAR with the bit resolution $B_{A D C}$ and the dynamic voltage range $V_{A D C}$ in order to obtain the discrete time form. The oversampling method with oversampling factor $m_{\text {over }}$ is also applied in order to reduce the effects of quantization noise in low bit quantization ADC [31]. After that, these components will be mixed again to form the symbols with double complex number. Then these symbols will go through SI channel estimation process to cancel SI signal.

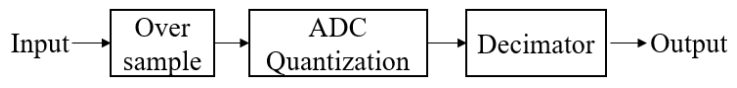

Fig. 4. ADC process.

\section{RESUlt AND Discussion}

\section{A. Simulation Specifications}

BER performances are computed by using Monte Carlo simulation on MATLAB. For 5G LDPC codes, the base graph
TABLE I

Simulation SPECIFICATIONS

\begin{tabular}{|l|l|}
\hline \multicolumn{1}{|c|}{ Parameter } & \multicolumn{1}{c|}{ Value } \\
\hline Codeword block size $(N)$ & 1024 bits \\
\hline Information size $(K)$ & 512 bits \\
\hline Code rate $(R)$ & $1 / 2$ \\
\hline Number of transmission frames & 1000000 \\
\hline CRC length & 11 \\
\hline Modulation scheme & QPSK \\
\hline Up-sample factor $\left(m_{u p}\right)$ & 4 \\
\hline Oversampling factor $\left(m_{\text {over }}\right)$ & 4 \\
\hline Quantization resolution in bits $\left(B_{D A C}, B_{A D C}\right)$ & $1,2,3,4,6,8,12,14$ \\
\hline Dynamic voltage range DAC $\left(V_{D A C}\right)$ & $2 \mathrm{~V}$ \\
\hline Dynamic voltage range ADC $\left(V_{A D C}\right)$ & $4 \mathrm{~V}$ \\
\hline SI channel taps & 3 \\
\hline Intended channel taps & 4 \\
\hline Forget factor $\lambda$ in RLS algorithm & 0.999 \\
\hline
\end{tabular}

matrix BG1 is implemented for all simulations. For 5G Polar codes, the SCLD-CA decoder technique with the list size $M=8$ is used. Moreover, BER performances of the FD transmission system are also evaluated by changing the value of quantization bit of DAC/ADC from 1 to 14 bits. Difference quantizer device architectures such as $\Sigma \Delta$, Gaussian with mid-rise, pipeline and SAR are applied in the ADC process at the receiver side. Low-bit ADC quantizers with oversampling method are also considered for low cost, low power consumption and simple hardware devices, which are the characteristics of green communications and IoT applications [31]. The simulation parameters are sum up in Table I.

\section{B. Effect of quantization noise and SI channel power}

First of all, the presence of DAC/ADC quantization noise has a significant impact on the channel estimation process, regardless of the channel coding used. As shown Fig. 5, it destroys from about 2 to $7 \mathrm{~dB}$ when SI channel power $p$ varies from 0 to $30 \mathrm{~dB}$, respectively, in SI channel estimation error when compared with the ideal case. Secondly, the SI channel power $p$ is also a major factor that impacts the system performance. Indeed, when $p$ increases, BER will decrease as shown in Fig. 6. In FD transmission with DSIC cancellation, when $p$ equal to $0 \mathrm{~dB}$ and $10 \mathrm{~dB}$, the results are closed to the case of HD transmission without SI happens. However, at high value of $p$, SI component becomes dominant factor and the curves levels off at higher BER values, so the system needs more power in SNR $(\mathrm{dB})$, defined by $p_{B} / \sigma_{w}^{2}$ with $p_{B}$ the transmitter power, to obtain the same result when $p$ increases to $20 \mathrm{~dB}$ and $30 \mathrm{~dB}$. In FD transmission without DSIC process, the system cannot estimate and reconstruct again the transmission signal.

\section{Effect of the bit resolution of DAC}

Next, BER performances of FD system are computed by changing the bit resolution $B_{D A C}$ of DAC for all channel coding schemes. While the 12 bits $\Sigma \Delta$ ADC will be set up at the receiver side with oversampling factor $m_{\text {over }}=4$. Based on the results in Fig. 7, we can observe that the quantization bit resolution of DAC affects significantly to the system performance. For instance, values of $B_{D A C}$ from 1 to 


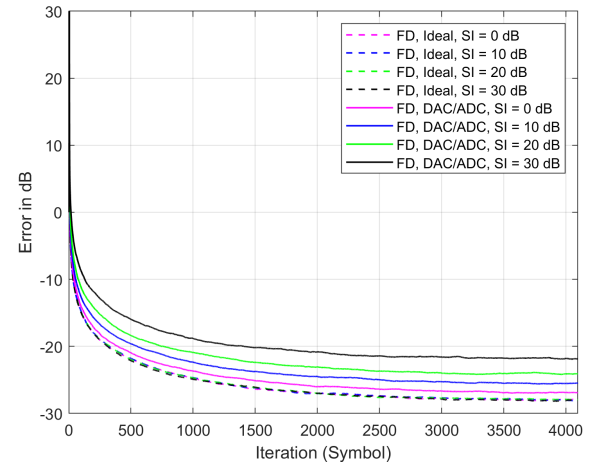

Fig. 5. SI channel estimation error of FD transmission with and without DAC/ADC process, $B_{D A C}=14$ bits, $B_{A D C}=12$ bits, $\Sigma \Delta$ ADC.

4 bits show the worst results than the others. In contrast, when values of $B_{D A C}$ goes to 6 bits and further, the system will obtain the saturation and reach nearly to the ideal case.

\section{Effect of the bit resolution of $A D C$}

Then, Fig. 8 shows the BER performances of FD system by changing the bit resolution $B_{A D C}$ of 4 different types of ADC, and oversampling factor $m_{\text {over }}=4$. The 5G LDPC channel coding scheme technique is used to illustrate, $p=30 \mathrm{~dB}$ and $B_{D A C}=14$ bits. For high bit resolution values from 6 bits to 14 bits, the system can approach nearly to ideal case. However, with the low-bit quantization from 1 to 4 bits ADC, the system needs more power $(\mathrm{dB})$ in SNR to obtain the same BER result with the ideal case. Compared with Subsection III-C, the lowbit ADC has better performances and converges quickly to free error because oversampling method has been applied in ADC process. Indeed, oversampling spreads the quantization noise in high frequency to reduce its effects. The gain between lowbit $\mathrm{ADC}$ and the ideal case is about $1 \mathrm{~dB}$ to $1.5 \mathrm{~dB}$ depends on ADC quantizer device architectures.

\section{E. Effect of ADC quantizer device architectures}

The quality of system performances also depends on the choice of different types of ADC quantizer such as $\Sigma \Delta$, Gaussian with mid-rise, pipeline as well as SAR. For all channel coding schemes, the system can reach approximately to the BER value of the ideal case for all of ADC quantizer types as illustrated in Fig. 9. Indeed, the $\Sigma \Delta$ is seemed to give a best result because the larger value of over-sampling factor, i.e, $m_{\text {over }}=4$ will give the better achievement in $\Sigma \Delta$ quantizer when compared with other ADC quantizer devices architectures, which has been proved in [31].

\section{F. Effect of channel coding schemes in FD transmission}

The effect of residual SI and quantization noise to the SI channel estimation process has been pointed out in Subsection III-B. To overcome this problem, channel coding schemes have been considered in FD transmission. Fig. 10 shows how channel coding schemes improve the BER performances in FD transmission system. It shows an interesting result where the system with channel coding can reconstruct well the information signal and it reaches approximately to the curve of ideal case. In contrast, in the case of FD transmission without channel coding, the gap between the curves of ideal case and the curves of DAC/ADC case are larger and can be observed clearly with the increasing of SNR. In HD transmission, the difference between ideal case and DAC/ADC case is less efficient, the system can estimate the information signal well when SI interference does not happen. While it cannot estimate the input information when the interference is not cancelled by DSIC process in FD transmission. Among these coding techniques, the 5G LDPC codes and LTE Turbo codes give quite similarity results, while Polar codes goes faster to free error and provides better performances although it needs more than $3 \mathrm{~dB}$ in SNR to go below the curve of the uncoding system. Consequently, it can be concluded that the channel coding schemes play an important role in the performance of FD transmission, it can compensate the influences of residual $\mathrm{SI}$ and quantization noise in the DAC/ADC process.

\section{G. Effect of choosing bit resolution of DAC/ADC}

Last but not least, the bit resolution in both DAC and ADC process is reduced to propose a trade-off in choosing the suitable bit resolution for a real communication system. The results are shown in Fig. 11. For all coding schemes, when the bit resolution in DAC/ADC process goes larger than 6 bits, the BER curves achieve nearly to the ideal case. However, when the bit resolution reduces to 4 bits or smaller, it notices that the bit resolution strongly affects significantly to the system performance. Therefore, combined with subsections III-C and III-D, the bit resolution should be chosen carefully depending on the applications. It is necessary to select the bit resolution of DAC/ADC from 6 bits and higher. Nevertheless, if we want to choose low-bit ADC from 1 to 4 bits at the receiver for green communication systems with simple hardware, low cost and low power consumption, the bit resolution of DAC must be set up at high values, i.e larger than 6 bits and oversampling method also needs to be applied in ADC process.

\section{CONCLUSION}

This paper points out the effects of residual SI and quantization noise due to the DAC/ADC process to the FD transmission system. Firstly, SI power is a major factor that degrades the system performances and it can be cancelled by using the DSIC process in FD transmission. Moreover, the quantization noise destroys and degrades significantly the transmission quality. Therefore, the bit resolution should be chosen carefully with high value, i.e, larger than 6 bits for both DAC/ADC process. If the green communications system and IoT applications are considered with low-bit ADC, i.e, from 1 to 4 bits, the bit resolution of DAC must be chosen at high value and the oversampling should be applied in ADC process in order to reduce the effects of quantization noise. Besides, the $\Sigma \Delta$ ADC seems to be the best choice in ADC process. Furthermore, the use of channel coding schemes plays a significant role in FD transmission. It can estimate and reconstruct the information signal approximately to the ideal case. Therefore, channel coding techniques such as 5G LDPC, Polar codes and Turbo codes are the potential candidates for 


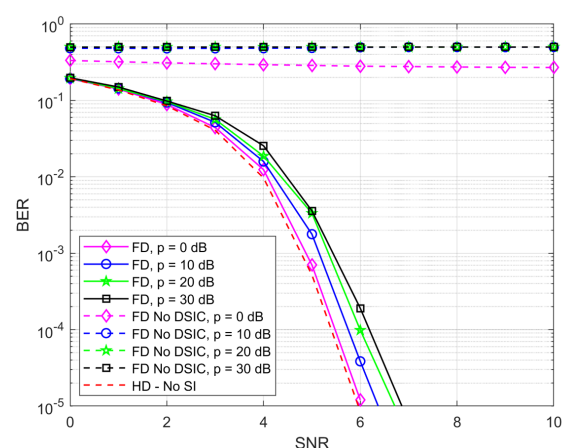

(a) LDPC

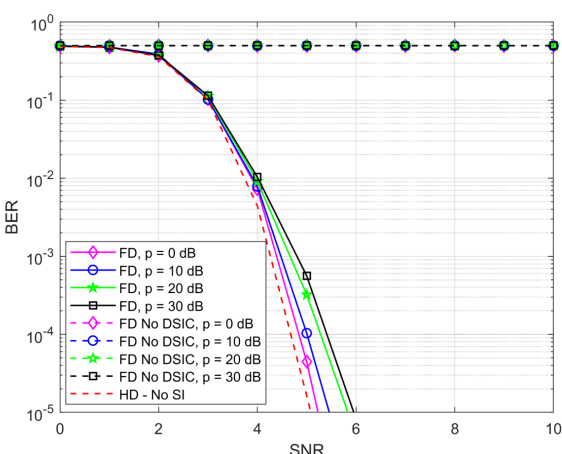

(b)Polar

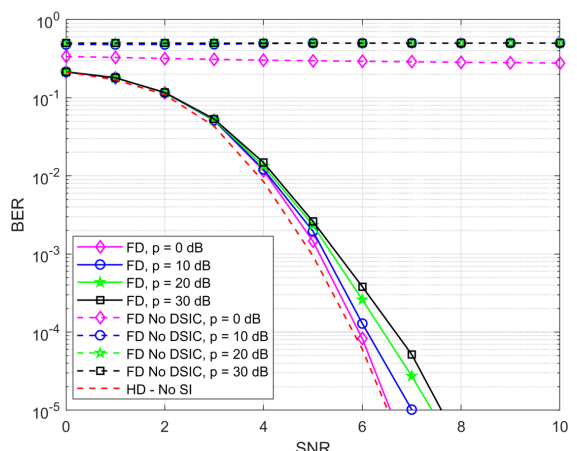

(c) Turbo

Fig. 6. BER of FD system with and without DSIC in different values of $p, B_{D A C}=14$ bits, $B_{A D C}=12$ bits, $\Sigma \Delta$ ADC.

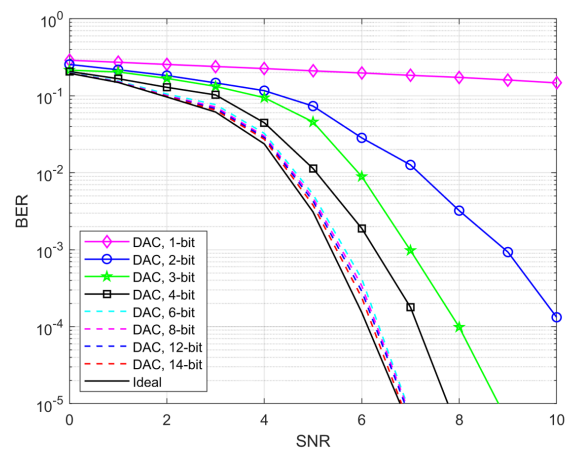

(a) LDPC

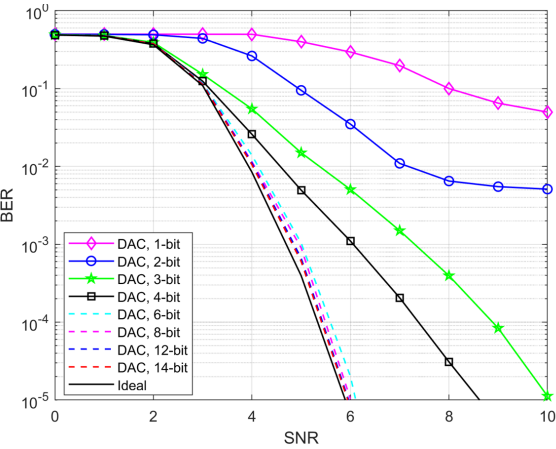

(b)Polar

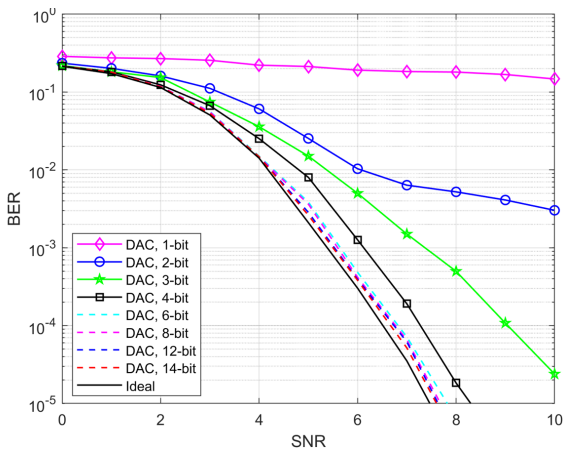

(c) Turbo

Fig. 7. BER of FD transmission with 12 bits $\Sigma \Delta \mathrm{ADC}$ and changing $B_{D A C}, p=30 \mathrm{~dB}$

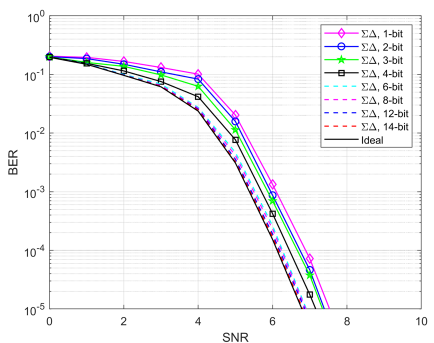

(a) $\sum \Delta$

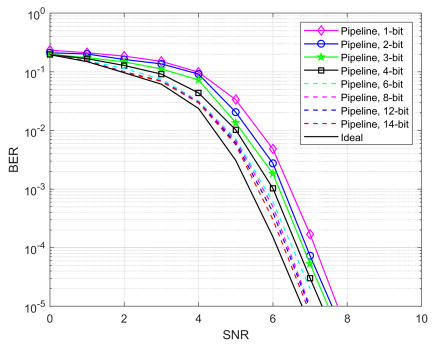

(c) Pipeline

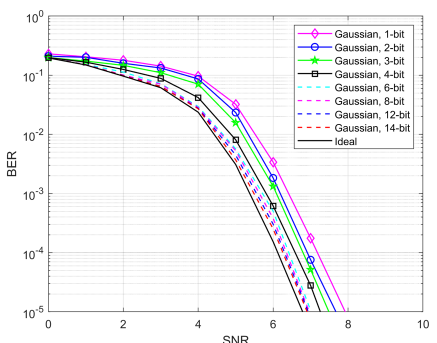

(b) Gaussian with mid-rise

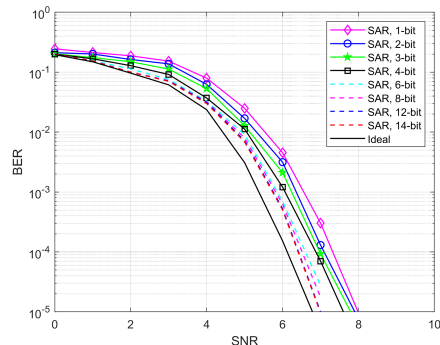

(d) SAR
Fig. 8. BER of FD transmission with $B_{D A C}=14$ bits and changing $B_{A D C}$.

the integrity of information in FD transmission, and 5G LDPC codes seem to give the best results.

\section{ACKNOWLEDGMENT}

This research is jointly supported by the IBNM (Brest Institute of Computer Science and Mathematics) CyberIoT Chair of Excellence at the University of Brest, and the Brittany region- Pôle d'Excellence Cyber.

\section{REFERENCES}

[1] B. V. Nguyen, H. Jung, and K. Kim, "Physical layer security schemes for full-duplex cooperative systems: State of the art and beyond," IEEE Communications Magazine, vol. 56, no. 11, pp. 131-137, 2018.

[2] M. Atallah, G. Kaddoum, and L. Kong, "A survey on cooperative jamming applied to physical layer security," in Proceedings of the IEEE International Conference on Ubiquitous Wireless Broadband, 2015.

[3] F. Zhu, F. Gao, T. Zhang, K. Sun, and M. Yao, "Physical-Layer Security for Full Duplex Communications With Self-Interference Mitigation," IEEE Transactions on Wireless Communications, vol. 15, no. 1, pp. 329340, jan 2016.

[4] E. Everett, A. Sahai, and A. Sabharwal, "Passive Self-Interference Suppression for Full-Duplex Infrastructure Nodes," IEEE Transactions on Wireless Communications, vol. 13, no. 2, pp. 680-694, feb 2014.

[5] E. Ahmed and A. M. Eltawil, "All-Digital Self-Interference Cancellation Technique for Full-Duplex Systems," IEEE Transactions on Wireless Communications, vol. 14, no. 7, pp. 3519-3532, 2015.

[6] Y. Wu, A. Khisti, C. Xiao, G. Caire, K. K. Wong, and X. Gao, "A Survey of Physical Layer Security Techniques for 5G Wireless Networks and Challenges Ahead," IEEE Journal on Selected Areas in Communications, vol. 36, no. 4, pp. $679-695,2018$.

[7] R. G. Gallager, "Low density parity check codes," IRE Trans. Inform. Theory, vol. IT-8, pp. 21-28, 1962.

[8] E. Arikan, "Channel polarization: A method for constructing capacityachieving codes for symmetric binary-input memoryless channels," IEEE Transactions on Information Theory, vol. 55, no. 7, 2009.

[9] C. Berrou, A. Glavieux, and P. Thitimajshima, "Near SHANNON limit error-correcting coding and encoding: Turbo-codes," in Proceedings of the IEEE International Conference on Communications, 1993.

[10] J. H. Bae, A. Abotabl, H. P. Lin, K. B. Song, and J. Lee, "An overview of channel coding for 5G NR cellular communications," Transactions on Signal and Information Processing, vol. 8, no. 17, 2019.

[11] 3GPP, "Release 16 - Multiplexing and channel coding," 2020

[12] O. Iscan, D. Lentner, and W. Xu, "A comparison of channel coding schemes for $5 \mathrm{~g}$ short message transmission," in The IEEE Globecom Workshops, 2016, pp. 1-6. 


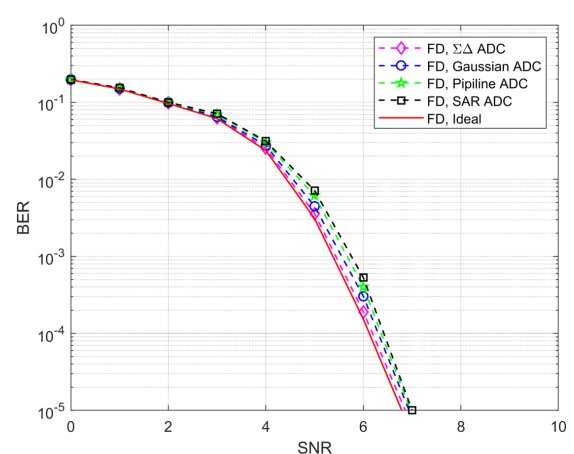

(a) LDPC

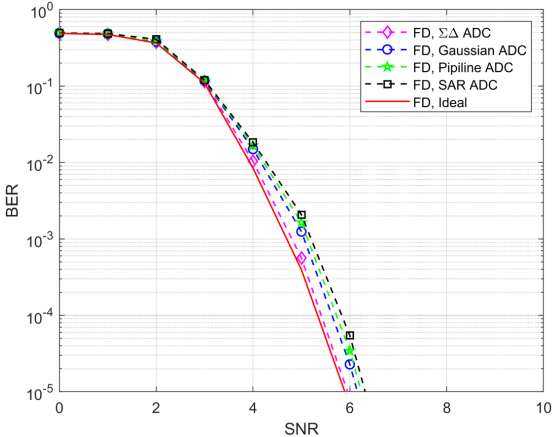

(b)Polar

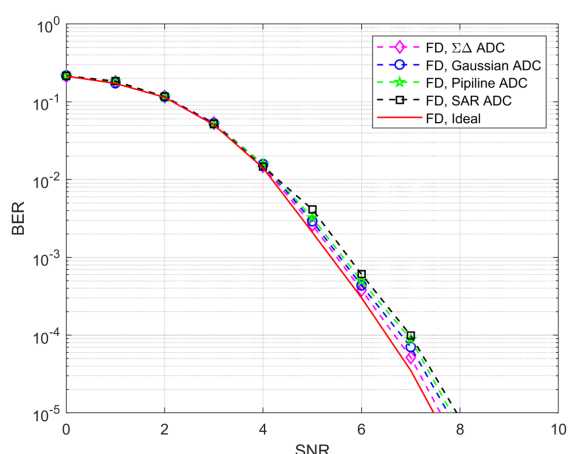

(c) Turbo

Fig. 9. BER of FD transmission with different types of ADC quantizer, $B_{D A C}=14$ bits, $B_{A D C}=12$ bits, $p=30 \mathrm{~dB}$.

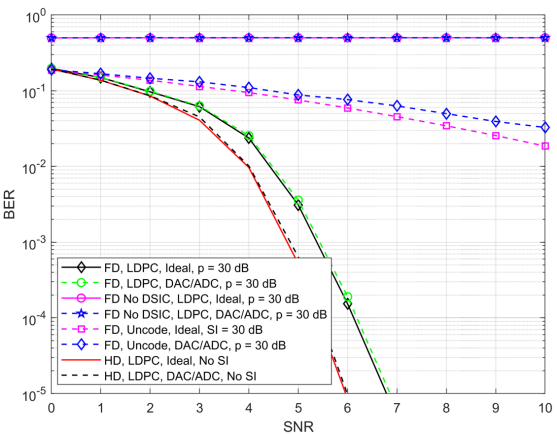

(a) LDPC

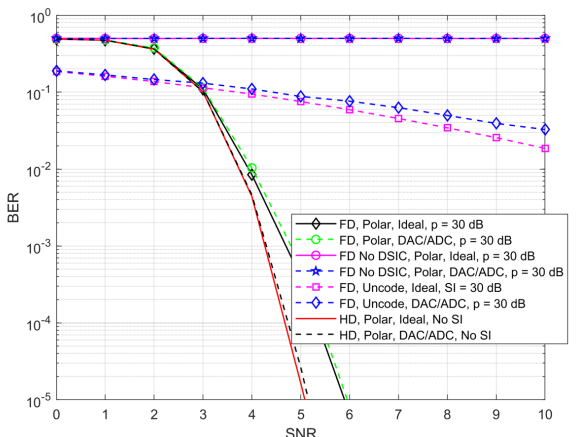

(b)Polar

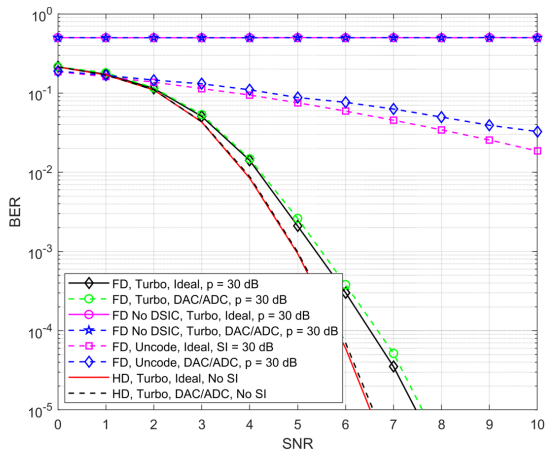

(c) Turbo

Fig. 10. Comparison between the FD system with and without DAC/ADC process, $B_{D A C}=14$ bits, $B_{A D C}=12$ bits, $\Sigma \Delta$ ADC.

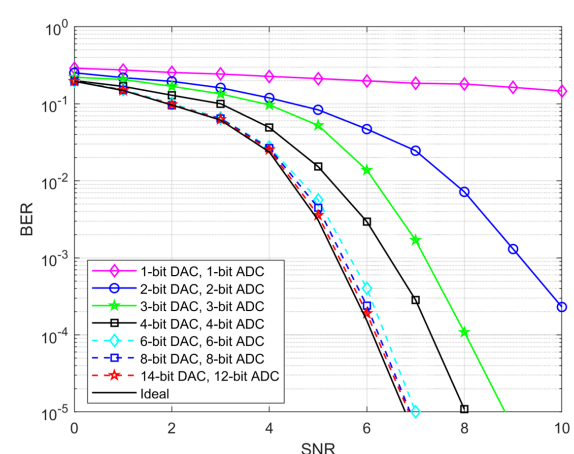

(a) LDPC

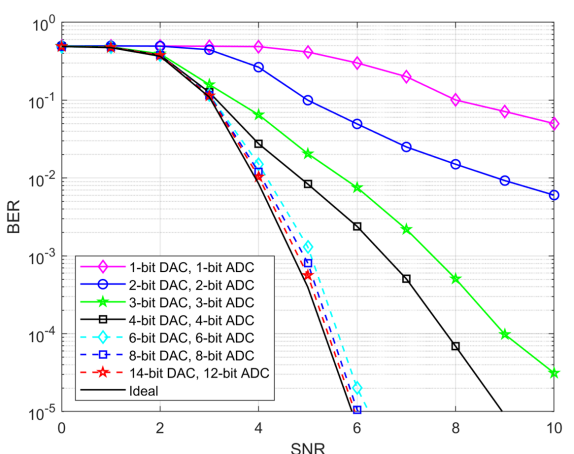

(b)Polar

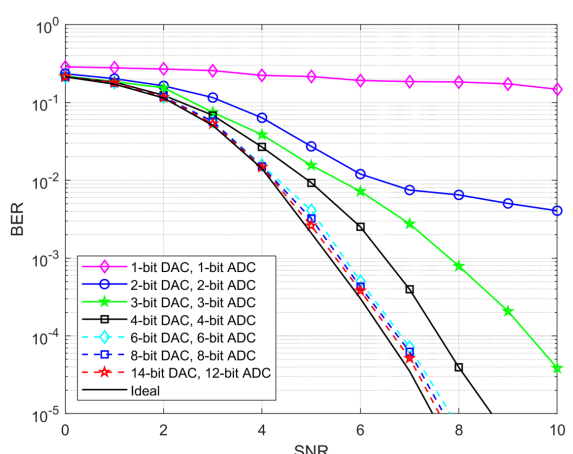

(c) Turbo

Fig. 11. BER of FD system with different bit resolution in DAC/ADC, $\Sigma \Delta \mathrm{ADC}, p=30 \mathrm{~dB}, m_{\text {over }}=4$.

[13] A. Masmoudi and T. Le-Ngoc, "Residual self-interference after cancellation in full-duplex systems," in 2014 IEEE International Conference on Communications (ICC), 2014, pp. 4680-4685.

[14] LTC2000 Family DAC Datasheet, Analog Devices.

[15] 12-Bit, 6 GSPS/10.25 GSPS, JESD204B, RF Analog-to-Digital Converter Datasheet, Analog Devices.

[16] C. Despina-Stoian, A. Digulescu-Popescu, S. Alexandra, R. Youssef, and E. Radoi, "Comparison of adaptive filtering strategies for selfinterference cancellation in lte communication systems," in The 13th International Conference on Communications (COMM), 2020.

[17] W. Kester, ADC Architectures III: Sigma-Delta ADC Basics Tutorial, Analog Devices, 2016.

[18] J. Max, "Quantizing for minimum distortion," IRE Trans. Inf. Theory, vol. 6, no. 1, pp. 7-12, 1960.

[19] W. Kester, ADC Architectures V: Pipelined Subranging ADCs Tutorial, Analog Devices, 2016.

[20] W.Kester, ADC Architectures II: Successive Approximation ADCs Tutorial, Analog Devices, 2009.

[21] A. Koohian, H. Mehrpouyan, A. A. Nasir, S. Durrani, and S. D. Blostein, "Residual self-interference cancellation and data detection in full-duplex communication systems," in 2017 IEEE International Conference on Communications (ICC).
[22] Guidelines for evaluation of radio transmission technologies for IMT2000, International Telecommunication Union, 1997.

[23] S. Haykin, Adaptive filter theory. Pearson, 1993, vol. 29.

[24] A. H. SAYED, Adaptive Filters. A John Wiley \& Song INC, 2008.

[25] 3GPP, "TS 38.212 NR- Multiplexing and channel coding," 2018.

[26] V. Bioglio, C. Condo, and I. Land, "Design of Polar Codes in 5G New Radio," IEEE Communications Surveys and Tutorials, 2020.

[27] X. Zhang and P. H. Siegel, "Quantized iterative message passing decoders with low error floor for LDPC codes," IEEE Transactions on Communications, vol. 62, no. 1, pp. 1-14, 2014.

[28] E. Sharon, S. Litsyn, and J. Goldberger, "An efficient message-passing schedule for LDPC decoding," in Proceedings of the IEEE Convention of Electrical and Electronics Engineers in Israel, no. 4, 2004.

[29] I. Tal and A. Vardy, "List Decoding of Polar Codes," IEEE Transactions on Information Theory, vol. 61, no. 5, pp. 2213-2226, 2015.

[30] S. Benedetto, G. Montorsi, F. Pollara, and D. Divsalar, "A Soft-Input Soft-Output Maximum A Posteriori (MAP) Module to Decode Parallel and Serial Concatenated Codes." TDA Progress Report, 1996.

[31] L. L. Nguyen, D. H. Nguyen, A. Fiche, T. Huynh, and R. Gautier, "Low-bit quantization methods for modulated wideband converter compressed sensing," in Proceedings of the IEEE Global Communications Conference, GLOBECOM, 2019. 